\title{
A familial clustering of autoimmune and monogenic diabetes
}

\author{
ANAND VELUSAMY, PAUL GRANT, KATE MOREL, NICK VAUGHAN
}

\begin{abstract}
Background: Monogenic diabetes type $\mathbf{5}$ or Renal Cysts And Diabetes (RCAD) is a familial syndrome associated with renal disease and diabetes, caused by mutations in the HNF-1B gene. Early recognition is important to plan appropriate treatment and specialist input.

Clinical case: We report the overlapping occurrence of type 1 diabetes and renal disease among different members of the same family. The aetiology of diabetes was autoimmune with strong antibody positivity, whilst the renal disease was caused by a mutation in the hepatocyte nuclear factor (HNF-1B) gene.

Conclusions: A low threshold of clinical suspicion is important to recognise monogenic forms of diabetes at an early stage. Mild renal impairment is usually asymptomatic in the initial phases and, as such, this mutation is likely to be underdiagnosed. An uncertain aetiology with multiple family members being affected at an early age should prompt the clinician to pursue further investigations.
\end{abstract}

Key words: MODY, RCAD, monogenic diabetes, type 1 diabetes, HNF1-beta

\section{Introduction}

The last few decades have seen a remarkable advancement in the understanding and diagnosis of a heterogeneous group of patients with monogenic diabetes (previously known as Maturity Onset Diabetes of the Young [MODY]).

The prevalence of monogenic diabetes is thought to be around $3 \%$ of the overall diabetes population, but it is frequently under diagnosed and its prevalence varies according to location. ${ }^{1-3}$ It is caused by a single gene defect affecting pancreatic beta-cell function, and it tends to run in families as it is inherited in an autosomal dominant fashion. Several types of monogenic diabetes have been identified and there is much ongoing research exploring the molecular pathogenesis. ${ }^{4}$

\section{Clinical case}

A 38 year-old Caucasian woman (KK) was diagnosed with type

Department of Diabetes, Royal Sussex County Hospital, Brighton, UK

Address for correspondence: Dr Paul Grant

E-mail: drpaul.grant@doctors.org.uk

Br J Diabetes Vasc Dis 2015;15:139-141

http://dx.doi.org/10.15277/bjdvd.2015.034
1 diabetes at the age of 5 years, which was later confirmed by a strong positivity for anti-GAD (titre $>2000 \mathrm{U} / \mathrm{mL}$ ) and anti-IA2 antibodies. She also had autoimmune hypothyroidism with positive anti-TPO antibodies (>500 mU/L), as did her father.

The patient's sister (RB) was diagnosed with type 1 diabetes relatively recently, at 39 years of age. She tested strongly positive for anti-GAD, anti-IA2 and anti-TPO antibodies. RB was known to have a pre-existing diagnosis of renal impairment, with an estimated glomerular filtration rate of $40 \mathrm{~mL} / \mathrm{min}$ and significant proteinuria, five years prior to the diagnosis of diabetes, although no clear diagnosis had been made as to the underlying cause of this. Notably, she did not have any other microvascular complications, such as diabetic retinopathy.

RB gave birth to a daughter (AB) now aged 6 years, who was born with a degree of renal impairment; subsequent ultrasound revealed a multicystic dysplastic right kidney and a left renal cyst with nephrocalcinosis.

Given the combination of familial diabetes and renal impairment and cysts developing at a young age, the possibility of a genetic problem was raised. Genome screening and mutation analysis confirmed the presence of a heterozygous hepatocyte nuclear factor (HNF-1B) mutation on exon 4 in patient RB, which would be consistent with monogenic diabetes type 5 (also known as Renal Cysts And Diabetes [RCAD]).

This led to targeted screening of her other family members for the same mutation, as shown in the accompanying flow chart (Figure 1). Her mother (RB), her aunt the proband (KK) and her grandmother $(\mathrm{CH})$ were all found to have the same mutation. Interestingly, $\mathrm{CH}$ (the proband's mother) did not have diabetes but was diagnosed with stage 3 chronic kidney disease with no albuminuria, and she had a history of absence seizures since the age of 3 years (cerebral disorders are a further recognised complication of HNF-1B mutations). The rest of the family tree is also outlined in Figure 1.

\section{Discussion}

The diagnosis of monogenic diabetes is important as it determines the most appropriate treatment for patients and also helps to advise them about how their diabetes is likely to progress in the future, as well as planning for pregnancy. As it runs in families, it is beneficial to screen family members for diabetes and associated abnormalities. Advancements in our understanding of the human genome, and specifically the different variants of MODY and their underlying pathophysiology, can lead to greater recognition and management of conditions such as type 5 monogenic diabetes type or RCAD. Box 1 compares the distin- 
Figure 1. Flowchart/family tree

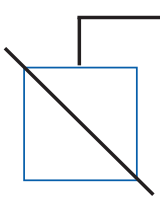

CKD3

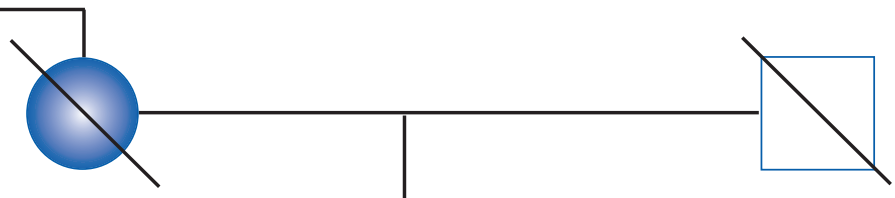

Diabetes ? type

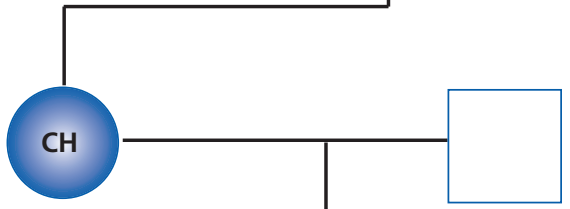

CKD3 diagnosed 2004

Childhood epilepsy

Autoimmune hypothyroidism

HNF-1B mutation

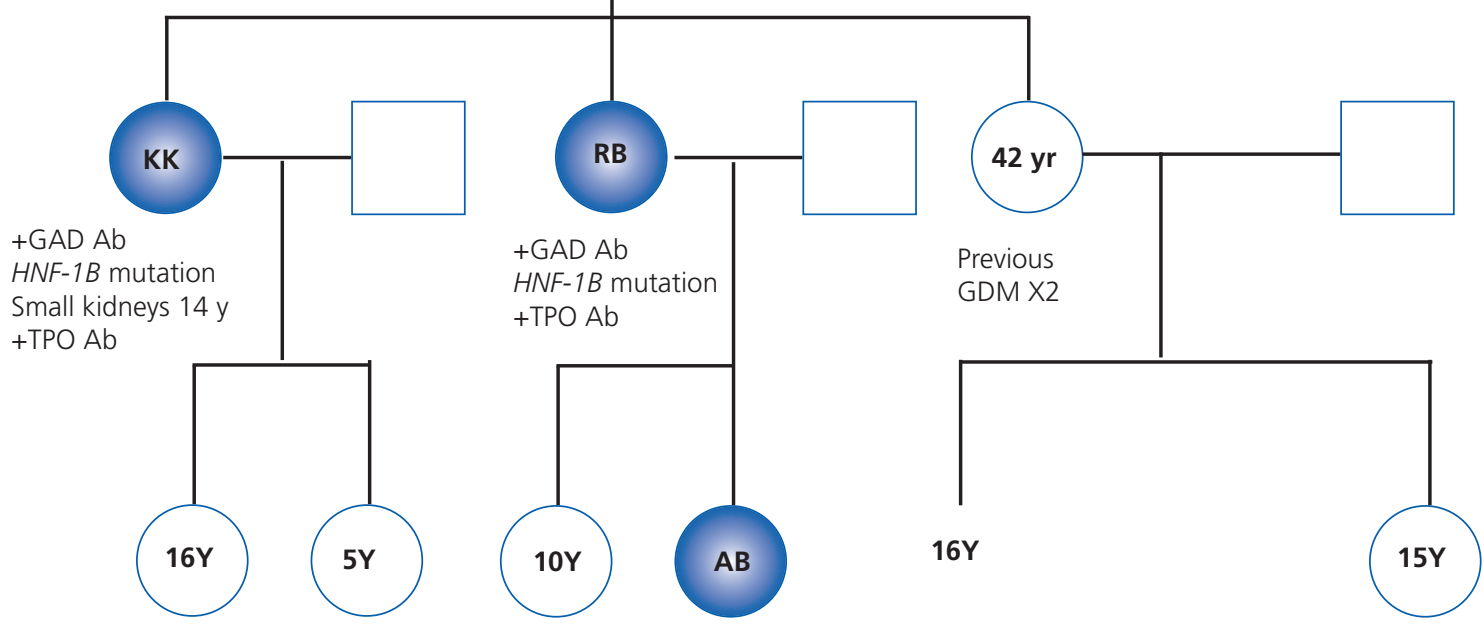

Healthy Healthy

Healthy

Congenital renal impairment; renal cysts; dyspraxia; lazy eye

HNF-1B mutation
Speech and language problems; global developmental delay; epilepsy; abnormal genitalia; lazy eye

Box 1. Features of monogenic diabetes and of monogenic diabetes type 5-9

Some distinguishing features of monogenic diabetes ${ }^{5-7}$

- $\quad$ Young age at diagnosis (typically $<25$ years, although patients can be older)

- Presence of endogenous insulin production

- Positive family history

- Negative for autoantibodies against beta-cell antigens

- Does not normally have features of insulin resistance

- $\quad$ Detectable C-peptide levels while on insulin after the expected honeymoon period (usually >3years)

- $\quad$ Other associated features (renal cysts, genital defects, myopathy, central nervous system defects)
Features of monogenic diabetes type 5 (RCAD/HNF-1B mutation) $)^{5,8,9}$

- Unexplained familial renal cystic disease

- $\quad$ Renal cysts may be detected in utero

- Pancreatic atrophy

- $\quad$ Early onset non-insulin dependent diabetes (25 years, wide spectrum of presentations from normal glucose tolerance to insulin-requiring diabetes)

- $\quad$ Genital tract malformations

- Hyperuricaemia and early onset gout

- Hypomagnesaemia

- Abnormal liver function tests 
guishing features of monogenic diabetes in general and monogenic diabetes type 5 in particular. ${ }^{5-9}$

In monogenic diabetes type 5, reduced insulin secretion is due to beta-cell dysfunction, which is related to pancreatic atrophy. ${ }^{10}$ The commonest renal abnormality is cystic disease (66\% of cases) although the most specific phenotype is familial hypoplastic glomerulocystic kidney disease.8,10,11 Renal cysts generally predate diabetes and can be found on fetal scans as early as 17 weeks. ${ }^{10-12}$

Although the mean age of diagnosis of monogenic diabetes type 5 is less than 30 years, it has been diagnosed as late as age 60 years. Our case report is a unique description of the coexistence of both type 1 diabetes with positive auto-antibodies and renal abnormalities associated with an HNF-1B mutation within the same family. The extent of the contribution of HNF-1B mutation to the burden of diabetes mellitus is difficult to quantify in this context as metabolic disturbance tends to occur at a later stage and with variable penetrance. Hence, it is very valuable to elicit a thorough family history for at least three generations with more focused probing on second and third degree relatives with diabetes.

\section{Conflict of interest None \\ Funding sources None}

Acknowledgements Genetic testing was conducted at the Centre for Molecular Genetics at the University of Exeter Medical School and Royal Devon and Exeter Hospital, Exeter. Further information is available from www.diabetesgenes.org.

\section{References}

1. Diabetes UK. State of the Nation. England. 2013. Available at https://www.diabetes.org.uk/Documents/About Us/What we say/0160b- state-nation-2013-england-1213.pdf

2. Kropff J, Selwood MP, McCarthy MI, et al. Prevalence of monogenic diabetes in young adults: a community-based, cross-sectional study in Oxfordshire, UK. Diabetologia 2011;54:1261-3. http://dx.doi.org/ 10.1007/s00125-011-2090-z

3. Ledermann HM. Maturity-onset diabetes of the young (MODY) at least ten times more common in Europe than previously assumed? Diabetologia 1995;38:1482. http://dx.doi.org/10.1007/BF00400611

4. McCarthy, MI, Hattersley, AT. Learning From Molecular Genetics. Novel insights arising from the definition of genes for monogenic and type 2 diabetes. Diabetes 2008;57:2889-98.

http://dx.doi.org/10.2337/db08-0343

5. Diabetes Genes, Diabetes Research Department and the Centre for Molecular Genetics, University of Exeter Medical School and Royal Devon and Exeter Hospital, Exeter. Genetic types of diabetes including maturity-onset diabetes of the young (MODY).

Available at http://diabetesgenes.org.

6. Jones AG, Hattersley AT. The clinical utility of C-peptide measurement in the care of patients with diabetes. Diabet Med 2013:30:803-17. http://dx.doi.org/10.1111/ dme.12159

7. Grant, P, Velusamy A, Turner E, Chakera A. When to suspect 'funny diabetes'. Clin Med 2014;14:663-6.

http://dx.doi.org/10.7861/clinmedicine.14-6-663

8. Faguer S, Decramer S, Chassaing N, et al. Diagnosis, management, and prognosis of HNF1B nephropathy in adulthood. Kidney Int 2011;80:768 76. http://dx.doi.org/10.1038/ki.2011.225

9. Bingham C, Ellard S, van't Hoff WG, et al. Atypical familial juvenile hyperuricemic nephropathy associated with a hepatocyte nuclear factor-1beta gene mutation. Kidney Int 2003;63:1645-51. http://dx.doi.org/10.1046/j.1523-1755.2003.00903.x

10. Bingham C, Hattersley AT. Renal cysts and diabetes syndrome resulting from mutations in hepatocyte nuclear factor-1 beta. Nephrol Dial Transplant 2004;19:2703-8. http://dx.doi.org/10.1093/ndt/gfh348

11. Edghill EL, Oram RA, Owens $M$, et al. Hepatocyte nuclear factor- 1 beta gene deletions-a common cause of renal disease. Nephrol Dial Tansplant 2008;23:627-35. http://dx.doi.org/10.1093/ndt/gfm603

12. Edghill EL, Stals K, Oram RA, et al. HNF1B deletions in patients with young-onset diabetes but no known renal disease. Diabet Med 2013;30:114-17. http://dx.doi.org/10.1111/j.1464-5491.2012.03709.x

\section{$A B C D$ \\ Exenatide weekly (QW) (Bydureon) Nationwide Audit in progress \\ Association of Eritish Clinical Diabetologists}

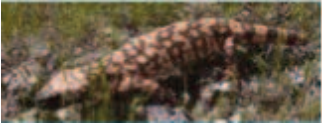

\section{Does your centre use exenatide QW?}

\section{If yes, REGISTER YOUR CENTRE! http://diabetologists-abcd.org.uk/n3/ExenatideQW_Audit.htm}

$A B C D$ has launched a nationwide audit of exenatide QW (Bydureon) in the UK to assess real clinical efficacy and safety \& inform future practice and guidelines

- you are invited to submit your patients' anonymised routinely collected data

- using an easy-to-use online tool hosted on the secure NHS network (N3)

- we can provide easy-to-complete paper proformas for use if preferred

- if contributing, you will be able to analyse your local data easily

Please remember: - the more data, the more significant the result will be - all contributors will be listed in publications arising from data submission 